\title{
Local Sampling Problems
}

\author{
Shou-Yuan Yang ${ }^{1}$ and Wei Lin ${ }^{2 \star}$ \\ 1 Department of Mathematics, Sun Yat-Sen University, \\ Guangzhou 510275, PR China \\ yshouy@263. net \\ 2 Department of Mathematics, Sun Yat-Sen University, \\ Guangzhou 510275, PR China \\ stslw@zsu.edu.cn
}

\begin{abstract}
The main purpose of this paper is to investigate the local error for the sampling problem in diverse situations. We find that the local error is heavily depending on the asymptotic behavior of the sampling function. By virtue of evaluating the decay of the sampling function, we give a local error estimation for uniform and non-uniform sampling in multiresolution analysis (MRA) and in shift-invariant spaces.
\end{abstract}

\section{Introduction and Preliminaries}

In digital signal and image processing and digital communications, a continuous function (signal) is usually represented by its discrete samples. Then how to reconstruct a continuous function from its discrete samples is a fundamental problem. Many mathematicians and engineers contribute to this area, e. g., see 1, 3, 13. Most of their work is mainly concerned with the whole domain cases, i.e., the sampling domain is the whole Euclidean space $\mathbb{R}^{d}$ and we want to recover the function on the whole space. But in many real applications, sampling may be limited to a bounded domain, and we need only to recover the function in a local area. So finding a suitable method to recover a function from its discrete samples locally is significant both in theory and application. The earliest results on local sampling may be found in 7]9]. In [2, N. Atreas et al. investigated the local error of the reconstruction formula in a multiresolution analysis (MRA). In 14, S. Y. Yang find a new method to evaluate the asymptotic rate of decay of the sampling function, and generalized their result to higher dimensional cases. In this paper, we shall investigate the local error for sampling problem in diverse situations, including sampling in shift-invariant spaces and in MRA, uniform sampling and non-uniform sampling.

\section{$1.1 \quad$ Notations}

Before proceeding with our discussion, we must introduce some notations first. $\mathbb{N}, \mathbb{Z}, \mathbb{R}, \mathbb{C}$ denote the set of natural numbers, integers, real numbers and complex numbers, respectively. $\mathbb{R}^{d}$ and $\Lambda$ denote the $d$-dimensional Euclidean space

\footnotetext{
* Two authors both supported by the Natural Science Fund of China and the Natural
} Science Fund of Guangdong province. 
and unit lattice respectively. $\mathbb{T}^{d}=\mathbb{R}^{d} / \Lambda$ is the $d$-dimensional torus. $l^{1}(\Lambda)$ denotes the well-known Banach space of all absolutely summable sequences on $\Lambda$. The Fourier transform of a function $f \in L^{1}\left(\mathbb{R}^{d}\right)$ is defined by

$$
\hat{f}(u)=\int_{\mathbb{R}^{d}} f(x) e^{-i 2 \pi\langle x, u\rangle} d x,
$$

this transform maps $s$ onto itself and extends to all tempered distributions $s^{\prime}$ by duality, here $s$ denotes the class of all infinitely differentiable functions with rapid decay at infinity. We shwll also use the Wiener Amalgam space $W\left(L^{p}\right)$ defined by

$$
W\left(L^{p}\right)=\left\{f \in L^{p}\left(\mathbb{R}^{d}\right):\|f\|_{W\left(L^{p}\right)}=\left(\sum_{k \in \Lambda} \sup _{x \in[0,1]^{d}}|f(x+k)|^{p}\right)^{1 / p}<\infty\right\},
$$

and we use $W_{0}\left(L^{p}\right)$ to denote its closed subspace of continuous functions. Endowed with the norm $\|\cdot\|_{W\left(L^{p}\right)}$, then $W\left(L^{p}\right)$ and $W_{0}\left(L^{p}\right)$ are Banach spaces; e.g., see [5] $6 V^{p}(\varphi)(1 \leq p \leq \infty)$ is the shift-invariant space defined by

$$
V^{p}(\varphi)=\left\{\sum_{k \in \Lambda} c(k) \varphi(\cdot-k): c \in l^{p}(\Lambda)\right\}
$$

where $\varphi$ is the so-called generator. For such a shift-invariant space to be well defined, we need the generator $\varphi \in W_{0}\left(L^{1}\right)$ and satisfies the following condition

$$
0<m \leq \sum_{k \in \Lambda}|\hat{\varphi}(\xi+k)|^{2} \leq M<\infty
$$

for some $m, M>0$. Under these conditions, the shift-invariant space $V^{p}(\varphi)$ is a closed subspace of $L^{p}\left(\mathbb{R}^{d}\right)(1 \leq p \leq \infty)$. Moreover, we have

$$
m_{p}\|c\|_{l^{p}(\Lambda)} \leq\left\|\sum_{k \in \Lambda} c(k) \varphi(\cdot-k)\right\|_{L^{p}\left(\mathbb{R}^{d}\right)} \leq M_{p}\|c\|_{l^{p}(\Lambda)}, \quad \forall c \in l^{p}(\Lambda)
$$

for $1 \leq p \leq \infty$ and some positive constants $m_{p}, M_{p}$ depending on $p$, e. g., see[1]. In this situation, the generator $\varphi$ is said to be stable. By the way, we also need the the following notation

$$
\hat{\varphi} *=\sum_{k \in \Lambda} \varphi(k) \exp (i 2 \pi\langle k, \cdot\rangle)
$$

defined on $\mathbb{T}^{d}$.

Multiresolution analysis (MRA) is a fundamental concept in wavelet theory; e.g., see 41112 .

Definition 1. A dyadic multiresolution analysis (MRA) of $L^{2}\left(\mathbb{R}^{d}\right)$ is a sequence $\left\{V_{m}: m \in \mathbb{Z}\right\}$ of closed subspaces of $L^{2}\left(\mathbb{R}^{d}\right)$ with the following properties: 
(i) $\forall m \in \mathbb{Z}, V_{m} \subseteq V_{m+1}$,

(ii) $\bigcap_{m} V_{m}=\{0\}, \overline{\bigcup_{m} V_{m}}=L^{2}\left(\mathbb{R}^{d}\right)$,

(iii) $f \in V_{m} \Leftrightarrow f\left(2^{-m} \cdot\right) \in V_{0}$,

(iv) $\exists \varphi \in V_{0}$, a scaling function, such that $\left\{\tau_{n} \varphi=\varphi(\cdot-n): n \in \Lambda\right\}$ is an orthonormal basis for $V_{0}$.

Throughout this paper we shall use the standard notation $\varphi_{m, n}=2^{m d / 2} \varphi\left(2^{m}\right.$. $-n)$ for $m \in \mathbb{Z}, n \in \Lambda$.

Definition 2. A closed subspace $V$ of $L^{2}\left(\mathbb{R}^{d}\right)$ is said to be a reproducing kernel Hilbert space (RKHS) with reproducing kernel $K(x, y)$ if and only if

$$
\forall f \in V, \quad x \in \mathbb{R}^{d}, \quad f(x)=\langle K(x, \cdot), f\rangle .
$$

Let $X=\left\{x_{j}: j \in J\right\} \subseteq \mathbb{R}^{d}$. Then $X$ is said to be separated if $\inf _{i \neq j}\left|x_{i}-x_{j}\right|>$ $0 ; X$ is said to be $\gamma_{0}$-dense in $\mathbb{R}^{d}$ if $\mathbb{R}^{d}=\bigcup_{j \in J} B_{\gamma}\left(x_{j}\right)$ holds for all $\gamma>\gamma_{0}$, where $B_{\gamma}\left(x_{j}\right)$ denotes the open ball centered at $x_{j}$ with diameter $\gamma$.

\subsection{Reconstruction Formula for Sampling Problems}

The basic idea of sampling is to recover a function $f(x)$ from its discrete samples $\left\{f\left(x_{j}\right): j \in J\right\}$. Of course, only special kind of functions can be reconstructed in this way, moreover, the sampling set $x=\left\{x_{j}: j \in J\right\}$ must satisfy certain density condition. For the functions in a shift-invariant space $V^{2}(\varphi)$, we can recover them from their samples on the unit lattice $\Lambda$. the following proposition is well-known (e. g., see 1314 ).

Proposition 1. Suppose the generator $\varphi \in W_{0}\left(L^{1}\right)$ is stable and $\hat{\varphi} *$ is nonvanishing on $\mathbb{T}^{d}$, where $\hat{\varphi} *$ is defined by (2) in subsection 1.1. Then

a. $\{\varphi(\cdot-n): n \in \Lambda\}$ is a Riesz basis for $V^{2}(\varphi)$;

b. $V^{2}(\varphi)$ is an RKHS with the reproducing kernel $K(x, y)$ determined by

$$
K(x, y)=\sum_{k \in \Lambda} \overline{\varphi(x-k)} \tilde{\varphi}(y-k)
$$

where $\tilde{\varphi}$ is the dual of $\varphi$ determined by

$$
\langle\varphi(\cdot-k), \tilde{\varphi}(\cdot-l)\rangle=\delta(k-l), \quad \forall k, l \in \Lambda ;
$$

c. There exist a unique function $s \in W_{0}\left(L^{1}\right)$ such that

$$
f=\sum_{k \in \Lambda} f(k) s(\cdot-k), \quad \forall f \in V^{2}(\varphi),
$$

$s$ is the so-called sampling function determined by

$$
\hat{s}=\hat{\varphi} / \hat{\varphi} * .
$$

For MRA cases, we also have the following well-known proposition, e. g., see[2]. 
Proposition 2. Let $\left\{V_{m}\right\}$ be an $M R A$ of $L^{2}\left(\mathbb{R}^{d}\right)$ with scaling function $\varphi \in$ $W_{0}\left(L^{1}\right)$. If $\varphi$ is stable and $\hat{\varphi} *$ is non-vanishing on $\mathbb{T}^{d}$, then

a. For each $m, V_{m}$ is a RKHS, the corresponding reproducing kernel $K_{m}$ is uniquely determined by

$$
K_{m}(x, y)=\sum_{n \in \Lambda} \overline{\varphi_{m, n}(x)} \varphi_{m, n}(y)
$$

b. There is a unique function $s \in V_{0}$ with the property that

$$
f=\frac{1}{2^{d m / 2}} \sum_{n \in \Lambda} f\left(\frac{n}{2^{m}}\right) s_{m, n}, \quad \forall f \in V_{m}
$$

the convergence is in $L^{2}\left(\mathbb{R}^{d}\right)$ and is absolute, and therefore also uniform on $\mathbb{R}^{d}$. Where $s$ is the so-called sampling function determined by (6) in Proposition [1]

For non-uniform sampling, we need the sampling set $X$ satisfy certain density condition. The following proposition are borrowed from [1].

Proposition 3. Suppose $\varphi \in W_{0}\left(L^{1}\right)$ is a stable generator, $X=\left\{x_{j}: j \in\right.$ $J\} \subseteq \mathbb{R}^{d}$ is a separated set, $K(x, y)$ is the reproducing kernel defined by (3) in Proposition 1. Then there exists some $\gamma_{0}>0$ such that if $X$ is $\gamma_{0}$-dense in $\mathbb{R}^{d}$, then $\left\{K\left(x_{j}, \cdot\right): j \in J\right\}$ is a frame for $V^{2}(\varphi)$. hence for any $f \in V^{2}(\varphi)$, we have the following reconstruction formula

$$
\left.\left.f(x)=\sum_{j \in J}\left\langle f, K\left(x_{j}, \cdot\right)\right\rangle \widetilde{K\left(x_{j}\right.}, \cdot\right)=\sum_{j \in J} f\left(x_{j}\right) \widetilde{K\left(x_{j}\right.}, \cdot\right),
$$

where $\left.\left\{\widetilde{\left(x_{j}\right.}, \cdot\right): j \in J\right\}$ is the dual frame of $\left\{K\left(x_{j}, \cdot\right): j \in J\right\}$. In this situation, $X$ is said to be a set of sampling

\subsection{Local Error for Sampling Problem}

Suppose we want to recover a function $f \in V^{2}(\varphi)$ in a bounded domain $\Omega \subseteq$ $\mathbb{R}^{d}$. Then we must sample in a neighborhood of $\Omega$. For simplicity, we suppose the sampling domain is $\Omega_{\delta}=\left\{x \in \mathbb{R}^{d}: \operatorname{dist}(x, \Omega)<\delta\right\}$, where $\operatorname{dist}(x, \Omega)=$ $\inf _{y \in \Omega}|x-y|$ denotes the distance between $x$ and $\Omega$, and we shall use $X_{\Omega, \delta}$ to denote the set $X \bigcap \Omega_{\delta}$.

Definition 3. Let $X$ be a set of sampling (see Proposition 3). Then the local error of the reconstruction formula (9) is defined by

$$
\left.E_{\Omega, \delta} f(x)=f(x)-\sum_{x_{j} \in X_{\Omega, \delta}} f\left(x_{j}\right) K \widetilde{\left(x_{j}\right.}, x\right), \quad x \in \Omega
$$


Since the reconstruction formulas (51) and (8) are just special cases of the reconstruction formula (9), we can define the local error of these reconstruction formulas similarly, i.e.

$$
E_{\Omega, \delta} f(x)=f(x)-\sum_{n \in \Lambda \bigcap} f(n) s(x-n), \quad x \in \Omega
$$

for (5) and

$$
E_{\Omega, \delta}^{m} f(x)=f(x)-\sum_{n \in \Lambda: 2^{-m}} f \in \Omega_{\delta} f\left(\frac{n}{2^{m}}\right) s\left(2^{m} x-n\right), \quad x \in \Omega
$$

for (8).

In general, the localization of these reconstruction formulas can be measured by the asymptotic rate of decay of the local error $E_{\Omega, \delta}$ (or $E_{\Omega, \delta}^{m}$ ) as $\delta$ increases. If the local error decays very fast, then the reconstruction formula is well localized, and we can recover a function from its samples locally. But as we shall see, the asymptotic rate of decay of the local error is heavily depending on the asymptotic behavior of the sampling function $s(x)$ (in uniform case) or the dual frame $\left.\left\{K \widetilde{\left(x_{j}\right.}, \cdot\right): j \in J\right\}$ (in non-uniform case), so we must evaluate the asymptotic rate of decay of the aforementioned sampling function and dual frame first. Fortunately, we have already many results on this topic at hand.

Here is a brief outline of this paper. In section 2, we evaluate the local error for uniform sampling problems, Theorem 1 Theorem 2, Theorem 3 and Theorem 4 are the main results in this section; In section 3, we evaluate the local error for the non-uniform sampling problem, Theorem 5 is the main result in this section.

\section{Local Error Estimation for Uniform Sampling}

This section mainly deals with the uniform case. We shall give several estimates for the local error in different situations. Through out this section, we always assume the generator (or scaling function in MRA case) $\varphi$ satisfies one of the following conditions

(C1) $\varphi(x)=O\left((1+|x|)^{-\beta}\right)$ as $|x| \longrightarrow \infty$ for some $\beta>\frac{d}{2}$;

(C2) $\varphi(x)=O(\exp (-\alpha \cdot|x|))$ as $|x| \longrightarrow \infty$ for some $\alpha>0$.

If the generator (or scaling function in MRA case) $\varphi$ satisfies condition (C1), then S. Y. Yang has already give the following result (see[14])

Theorem 1. Assume the generator $\varphi \in W_{0}\left(L^{1}\right)$ is stable and satisfies condition (C1) for some $\beta>\frac{d}{2}$. Then the local error $E_{\Omega, \delta} f(x)$ defined by [11) in subsection 1.3 satisfies

$$
\sup _{x \in \Omega}\left|E_{\Omega, \delta} f(x)\right|=O\left(\delta^{d / 2-\beta}\right) \cdot\|f\|_{L^{2}\left(\mathbb{R}^{d}\right)} \text { as } \delta \longrightarrow \infty, \quad \forall f \in V^{2}(\varphi) .
$$


Theorem 2. Assume the scaling function $\varphi \in W_{0}\left(L^{1}\right)$ is stable and satisfies condition (C2) for some $\beta>\frac{d}{2}$. Then the local error $E_{\Omega, \delta}^{m} f(x)$ defined by (12) in subsection 1.3 satisfies

$$
\sup _{x \in \Omega}\left|E_{\Omega, \delta}^{m} f(x)\right|=O\left(\delta^{d / 2-\beta}\right) \cdot\|f\|_{L^{2}\left(\mathbb{R}^{d}\right)} \text { as } \delta \longrightarrow \infty, \quad \forall f \in V_{m}(\varphi) .
$$

Now we assume the generator (or scaling function in MRA case) is continuous and satisfies condition (C2), then $\varphi$ automatically belongs to $W_{0}\left(L^{1}\right)$. We first give the following lemma, which will also be used in the next section, its proof is direct, one can see 15, Lemma 3.1.

Lemma 1. Let $I_{1}(\delta, \alpha)=\int_{|y| \geq \delta}|y|^{-d-\alpha} d y, I_{2}(\delta, \alpha)=\int_{|y| \geq \delta} \exp (-\alpha \cdot|y|) d y$, then we have

$$
I_{1}(\delta, \alpha)=2 \pi^{d / 2} \Gamma\left(\frac{d}{2}\right)^{-1} \cdot \frac{1}{\alpha} \delta^{-\alpha}
$$

and

$$
I_{2}(\delta, \alpha)=2 \pi^{d / 2} \Gamma\left(\frac{d}{2}\right)^{-1} \cdot e^{-\alpha \delta} \cdot\left(\sum_{k=0}^{d-1} \frac{(d-1) !}{(d-1-k) !} \cdot \alpha^{-k-1} \cdot \delta^{d-1-k}\right) .
$$

To evaluate the asymptotic rate of decay of the sampling function $s(x)$, we need the following lemma, its proof is found in 10 .

Lemma 2. Suppose $\varphi \in W_{0}\left(L^{1}\right)$ satisfies condition (C2) for some $\alpha>0$. a. If $\varphi$ is the generator of a shift-invariant space $V^{2}(\varphi)$, then the sampling function $s(x)$ determined by (6) in Proposition 1 satisfies

$$
s(x)=O\left(\exp \left(-\alpha^{\prime}|x|\right)\right) \text { as }|x| \longrightarrow \infty
$$

for some $\alpha^{\prime} \in(0, \alpha)$;

$b$. If $\varphi$ is the scaling function of an MRA, then the sampling function $s(x)$ determined by (6) also satisfies (17) for some $\alpha^{\prime} \in(0, \alpha)$.

Now we can evaluate the decay of the local error. The following theorem can be proved in the same way as Theorem 3.1 in 14 , so we omit its proof.

Theorem 3. Assume the generator $\varphi$ is continuous, stable and satisfies condition (C2) for some $\alpha>0$. Then for any $f \in V^{2}(\varphi)$, the local error $E_{\Omega, \delta} f(x)$ defined by (11) in subsection 1.3 satisfies

$$
\sup _{x \in \Omega}\left|E_{\Omega, \delta} f(x)\right|=O\left(\delta^{(d-1) / 2} \cdot \exp \left(-\alpha^{\prime} \delta\right)\right) \cdot\|f\|_{L^{2}\left(\mathbb{R}^{d}\right)} \text { as } \delta \longrightarrow \infty
$$

for some $\alpha^{\prime} \in(0, \alpha)$.

For the MRA case, similar result also holds. The following theorem can be proved in the same way as theorem 3, so we also omit its proof. 
Theorem 4. Assume the scaling function $\varphi$ is continuous, stable and satisfies condition (C2) for some $\alpha>0$. Then for any $f \in V^{2}(\varphi)$, the local error $E_{\Omega, \delta}^{m} f(x)$ defined by (12) in subsection 1.3 satisfies

$$
\sup _{x \in \Omega}\left|E_{\Omega, \delta}^{m} f(x)\right|=O\left(\delta^{(d-1) / 2} \cdot \exp \left(-\alpha^{\prime} \delta\right)\right) \cdot\|f\|_{L^{2}\left(\mathbb{R}^{d}\right)} \quad \text { as } \quad \delta \longrightarrow \infty
$$

for some $\alpha^{\prime} \in(0, \alpha)$.

\section{Local Error Estimation for Non-uniform Sampling}

This section mainly deals with the non-uniform case. Through out this section, we assume the generator $\varphi$ either satisfy condition (C2) defined in section 2 or satisfy the following condition

(C3) $\varphi(x)=O\left((1+|x|)^{-\beta}\right)$ as $|x| \longrightarrow \infty$ for some $\beta>d$.

Hence if moreover $\varphi$ is continuous, then in both cases we can conclude that $\varphi \in W_{0}\left(L^{1}\right)$. By Proposition 3 if $X$ is a set of sampling, then the reconstruction formula (9) holds. It is easy to observe that the localization of this reconstruction formula is determined by the off-center decay of the dual frame $\left.\left\{K \widetilde{\left(x_{j}\right.}, \cdot\right): j \in J\right\}$, because if each $\left.\widetilde{\left(x_{j}\right.}, \cdot\right)$ is concentrated in a neighborhood of $x_{j}$, then $f(x)$ is mainly determined by few samples which are near the point $x$.

So evaluating the off-center decay of every $\left.\widetilde{K\left(x_{j}\right.}, \cdot\right)$ is important. Fortunately, S. Jaffard investigated the off-diagonal decay of the infinite matrices, got the conclusion that the inverse matrix has almost the same off-diagonal decay as the original matrix (see[8]). Recently, K. Gröchenig using Jaffard's result to evaluate the off-center decay of the dual frame $\left\{K\left(\widetilde{\left(x_{j}\right.}, \cdot\right): j \in J\right\}$, e. g. see[10]. The following lemma is one of his main results.

Lemma 3. Assume the generator $\varphi$ is continuous and stable, $K(x, y)$ is the corresponding reproducing kernel defined by (3) in Proposition 1, $X=\left\{x_{j}: j \in\right.$ $J\}$ is a set of sampling, $\left.\left\{K \widetilde{\left(x_{j}\right.}, \cdot\right): j \in J\right\}$ is the dual frame of $\left\{K\left(x_{j}, \cdot\right): j \in J\right\}$. a. If moreover $\varphi$ satisfies condition (C3), then we have

$$
\left|K\left(x_{j}, y\right)\right| \leq C(X, \varphi)\left(1+\left|y-x_{j}\right|\right)^{-\beta}, \quad \forall j \in J,
$$

where $C(X, \varphi)$ is a constant depending on $X$ and $\varphi$, independent of $j$.

b. If moreover $\varphi$ satisfies condition (C2) for some $\alpha>0$, then there exists some $\alpha^{\prime} \in(0, \alpha)$ such that

$$
\left|K\left(x_{j}, y\right)\right| \leq C(X, \varphi) \exp \left(-\alpha^{\prime}\left|y-x_{j}\right|\right), \quad \forall j \in J
$$

where $C(X, \varphi)$ has the same property as in a.

By virtue of the above result, we now can give an evaluation for the local error $E_{\Omega, \delta} f(x)$ defined by (10) in subsection 1.3. 
Theorem 5. Suppose the generator $\varphi$ is continuous and stable, $X=\left\{x_{j}: j \in\right.$ $J\}$ is a set of sampling satisfying $\inf _{i \neq j}\left|x_{i}-x_{j}\right|=\mu>0$.

a. If moreover $\varphi$ satisfies condition (C3), then the local error $E_{\Omega, \delta} f(x)$ defined by (10) in subsection 1.3 satisfies

$$
\sup _{x \in \Omega}\left|E_{\Omega, \delta} f(x)\right|=O\left(\delta^{d / 2-\beta}\right) \cdot\|f\|_{L^{2}\left(\mathbb{R}^{d}\right)} \text { as } \delta \longrightarrow \infty, \quad \forall f \in V^{2}(\varphi) .
$$

b. If moreover $\varphi$ satisfies condition (C2) for some $\alpha>0$, then there exists some $\alpha^{\prime} \in(0, \alpha)$ such that

$$
\sup _{x \in \Omega}\left|E_{\Omega, \delta} f(x)\right|=O\left(\delta^{(d-1) / 2} \cdot \exp \left(-\alpha^{\prime} \delta\right)\right) \cdot\|f\|_{L^{2}\left(\mathbb{R}^{d}\right)} \text { as } \delta \longrightarrow \infty, \quad \forall f \in V^{2}(\varphi) \text {. }
$$

Because of page limit, we omit the proof of this theorem, but we will present it in another paper.

\section{References}

1. A. Aldroubi, and K. Gröchenig, Nonuniform sampling and reconstruction in shiftinvariant spaces. SIAM Rev. 43(4)(2001) 585-620

2. N. Atreas, J. J. Benedetto, and C. Karanikas, Local sampling for regular wavelet and Gabor expansions(to appear)

3. John J. Benedetto, Irregular sampling and frames, in: C. K. Chui (Ed.), Wavelets: A Tutorial in Theory and Applications. (1992) 445-507

4. I. Daubechies, Ten Lectures on Wavelets. CBMS-NSF Series in Applied Math. SIAM Philadelphia. 1992.

5. H. G. Feichtinger, Generalized amalgams, with application to Fourier transform. Cand. J. Math. 42(1990) 395-409

6. H. G. Feichtinger, Wiener amalgams over Euclidean spaces and some of their applications. In Proc. Conf. Function Spaces, K. Jarosz, ed., Lecture Notes in Math. 136, Springer-Verlag, New York, 1991, pp.123-137

7. H. D. Helms and J. B. Thomas, Truncation error of sampling theorem expansions. Proc. IRE 50(1962) 179-184

8. S. Jaffard, Propriétés des matrices "bien localisées" prés de leur diagonale et quelques applications. Ann. Inst. H. Poincaré Anal. Non Linéaire 7(5) (1990) 461476

9. D. Jagerman, Bounds for truncation error of the sampling expansions. SIAM J. Appl. Math. 14(1966) 714-723

10. K. Gröchenig, Localization of frames, Banach frames, and the invertibility of the frame operator. J. Fourier Anal. Appl.(to appear)

11. S. Mallat, A Wavelet Tour of Signal Processing. Academic Press, Boston, 1998

12. Y. Meyer, Ondelettes Et Opérateurs. Hermann, Paris, 1990

13. Michael Unser, Sampling-50 years after Shannon. Procdings of the IEE. 88 (4) (2000) 569-587

14. S. Y. Yang, Local error estimation for samling problems, Appl. Math. Comp.(to appear)

15. S. Y. Yang, The local property of several operators on sampling, Applicable Anal.(to appear)

16. K. Yao, Application of reproducing kernel Hilbert spaces-bandlimited signal models. Inform. and control. 11 (1967) 429-444 